\title{
How to Reduce the Main Risk of Menopausal Hormone Therapy (MHT, HRT, HT)
}

\author{
Xiangyan Ruan ${ }^{1,2}$ and Alfred 0 Mueck $^{1,2 *}$ \\ ${ }^{1}$ Department of Gynecological Endocrinology, Beijing Obstetrics and Gynecology Hospital, Capital Medical University, China \\ ${ }^{2}$ Department for Women's Health, University Women's Hospital and Research Center for Women's Health, Germany \\ *Corresponding author: Xiangyan Ruan, Department of Gynecological Endocrinology, Beijing Obstetrics and Gynecology Hospital, \\ Capital Medical University, Beijing 100026, China
}

To Cite This Article: Xiangyan Ruan, Alfred O Mueck, How to Reduce the Main Risk of Menopausal Hormone Therapy (MHT, HRT, HT). 2020 11(3). AJBSR.MS.ID.001623. DOI: 10.34297/AJBSR.2020.11.001623.

Received: 阱 December 09, 2020; Published: 制 December 16, 2020

\begin{abstract}
Most societies with interest in the field of estrogen/progestogen-therapy in peri- and postmenopausal women have now agreed to give this important area of gynecology the name "Menopausal Hormone Therapy" (MHT) instead of "hormone replacement therapy" (HRT) or "hormone therapy" (HT). The different terminology includes different assessments, different rationales, of this therapeutic area. There remains still some confusion since the definition of "menopause" is the "last period", but indeed this treatment is offered not only during the time of or after the last period but for example also is urgently needed for women with primary ovarian insufficiency (POI) (for example after chemotherapy) or after oophorectomy, i.e. during a long period in womens' life. Thus, the old term 'HRT' is also still in use, even the usual treatment of peri- and postmenopausal women should not be seen as a "replacement" (R), but rather as a treatment of symptoms, as this is considered using in the term "hormone therapy" (HT).

Asking "How to reduce the risk of this therapy" independent from this terminology it seems reasonable, to look at the dependency of risk on the two components of this therapy - the estrogen component, and the progestogen component. Thereby in hysterectomized women it is recommended to use only the estrogen therapy, which can reduce the four main risks of MHT (HRT, HT) which has been observed in the most important trial using MHT, the Women's Health Initiative (WHI): increased risk of venous thromboembolism, of stroke and coronary heart disease, and the risk of breast cancer. In the following in will be briefly discussed how these main risks could be reduced dependent on the two components of MHT.
\end{abstract}

\section{Estrogens: Which choice in MHT considering breast and cardiovascular system?}

In the WHI the estrogen component of MHT has been "conjugated equine estrogens" (CEE). This is a mixture of at least 10 different estrogenic components whereby about 30\% only are produced in horses, not in women. So, from the pharmacological view it is not really possible to predict the effect using CEE for MHT, because with 10 components, all biologically active, a clear doseefficacy relationship never can be shown. The individual variation of benefits and risks must vary in large extent, considering that using CEE not only 10 substances are working but also their amount in the tablets are different. We never can be sure what we give our patients with this form of estrogenic mixtures. In addition, we are not able to monitor the therapy by assessement of hormone levels, using 10 different estrogenic components. Therefore, especially in Europe for many years the physiological estrogen, the estradiol (E2), has been preferebly used in MHT and is now increasingly recommended also in other countries such as the USA or in our hospitals in China. In addition, for local vaginal treatment, also Estriol (E3) is in use, which, however, used properly, almost confer no systemic positive or negative effect and can be added in patients with vaginal complaints to the systemic treatment using CEE or E2.

Regarding the benefits of estrogens using as MHT like for treatment of climacteric symptoms, prevention of osteoporotic fractures, prevention of coronary heart disease (if the MHT is early started in perimenopause or early postmenopause), prevention of colon cancer, prevention of Alzheimer disease (if early started) 
there is no difference between the use of CEE and E2, if both given orally. Even a reduction of breast cancer, which has been shown with CEE in the WHI (estrogen-only arm) recently has been seen also using E2 in MHT. However, since E2 in contrast to CEE also can be used transdermally (patches, gel), there is a difference in the risk profile: the use of transdermal E2 can reduce the risk of venous thrombosis in contrast to all oral estrogens. So, in patients with increased risk of venous thrombosis (which according to own research is very rare in China in contrast to Western World!), the use of patches or gels delivering E2 through the skin directly in the systemic system urgently is recommended.

\section{Progestogens: Which choice in MHT considering breast, endometrium and cardiovascular system?}

The primary indication to use a progestogen in MHT is endometrial protection from unopposed estrogen therapy. Adequate progestogen addition urgently is recommended in women with intact uterus. Progestogen is not generally indicated with estrogen therapy post hysterectomy. Progestogens should have a high endometrial efficacy protecting the endometrium from estrogen induced hyperproliferation which could lead via hyperplasia to endometrial cancer. Clinically the addition to estrogen replacement should lead to regular bleeding pattern in sequential MHT and rapid amenorrhoe in continuous combined MHT.

In addition the most important criteria for the choice of a progestogen in MHT is tissue neutrality - no proliferation effects in the breast, neutral in the vasculature and in the metabolic system maintaining beneficial effects in lipids and glucose metabolism. Progesterone, the physiological progestogen, mostly meets those criteria, and also its retroisomer dydrogesterone, which has stronger endometrial efficacy. In contrast to various synthetic progestogens these more physiological progestogens do not further increase the estrogen induced risk of venous thromboembolism, they do not antagonize the cardiovascular benefit of estrogens, and they may not increase the risk of breast cancer at least up to 8 years treatment, derived from clinical data and main mechanisms of carcinogenicity. A recent Editorial of the journal "Menopause" suggested that the increase in breast risk in WHI could be explained by overexpression of certain membrane-bound steroid receptors. Own work showed that progesterone does not, in contrast to most synthetic progestogens. Other benefits of certain progestogens like the potency of drosperinone to maintain or even decrease the blood pressure can be considered at an individual basis, e.g. in cardiovascular risk patients, but are of secondary importance.

\section{MHT and the Breast}

Physicians and patients mostly fear the risk of breast cancer using MHT although the morbidity and mortality is less than $10 \%$ compared to the risk of cardiovascular diseases which is more than
50\%. The Women's Health Initiative (WHI) has been the only study for treatment of postmenopausal women with MHT performed within a double-blind placebo-controlled design, assessing clinical endpoints in large patient samples. In the estrogen-only arm for hysterectomized women CEE was used, in the combined arm MPA was added. For the combined MHT ( $\mathrm{n}=16,608$ ) a relative risk of breast cancer (BC) of 1.26 (95\% CI 1.00-1.59) was reported. No increased risk for women using CEE-only $(n=10,739)$ was seen, even a significant decrease in compliant women which persisted after stop of the study. Recently in a randomized open labeled control prospective study lasting 11 years (the Danish Osteoporosis Prevention, DOPS) also with the use of estradiol a significant reduction of breast cancer was seen (HR 0.54, CI 0.320.91), although the numbers in this estrogen-only group, used in hysterectomized women was small.

Obviously particularly the progestogen component may increase the risk of BC. Already extensively published experimental research performed at the Beijing Obstetrics \& Gynecology Hospital (Capital Medical University, China) in collaboration with the University Women's Hospital in Tuebingen, Germany, can explain those results, since carcino protective effects of estrogens like apoptosis can work, if the proliferation of BC cells is slow enough to destroy the cancer before its clinical detection. However, in case of fast proliferation, clinical cancer can develop. Progestogens can accelerate proliferation via special cell membrane-bound components (e.g. Progesterone Receptor Membrane Component-1; PGRMC1) which we detected in the tissue of patients with breast cancer and since very recently also can assess in the circulation as marker for an increased breast cancer risk. Future research may lead to the possibility to screen for patients at risk derived from those mechanism.

For the "total evidence" also observational studies should be considered for the choice of an optimal therapy. In about 20 studies using combined MHT an increased BC risk has been observed comparable with the WHI study. At this time only for the use of the natural progesterone or its retroisomer dydrogesterone no increase of BC risk or at least a significantly lower risk has been seen in observational studies in contrast to other tested synthetic progestogens like norethisterone or medroxyprogesterone acetate. However, certain patients may be of higher risk, and we hope to be able soon to identify those patients by screening for special risk factors in their breast tissue and also now in blood assessing new markers to predict breast cancer risk (like PGRMC1) and their hormonal profiles (search for genotoxic hormone metabolites and genetic polymorphisms of protective enzymes). Nevertheless, MHT with the best benefit/risk ratio should be used to minimize possible risks. 